\title{
L'Alimentation en eau potable de la ville d'Ankara nouvelle capitale de la Turquie
}

\author{
par F. A. Liefrinck, Ingéneur Civil \\ Institut du Gouvernement Néerlandais pour l'alimentation en eau potable, La Haye, Hollande
}

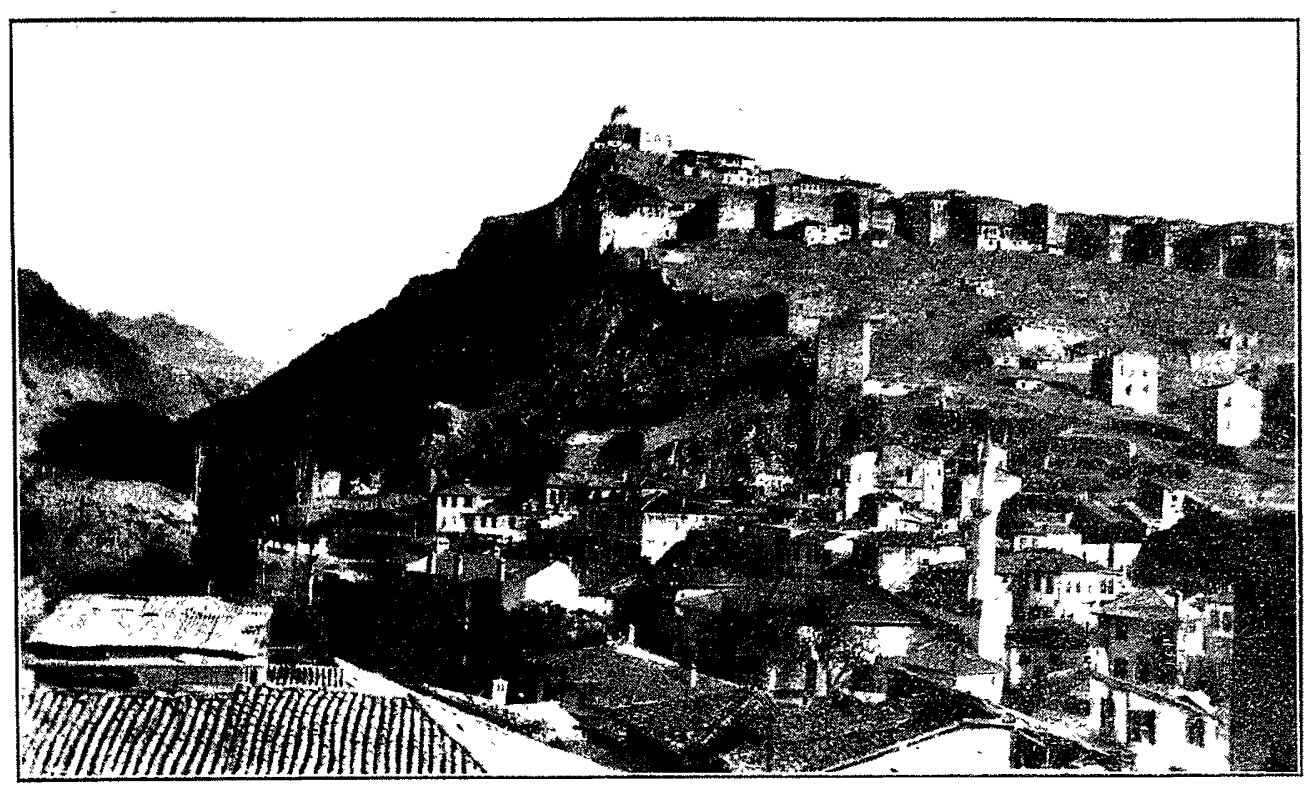

Fig. 1. - La citadelle.

Vingt siècles avant l'ère chrétienne - comme nous l'apprennent les archéologues - les anciens Hittites s'établirent à Ancyra (Angora), au cœur des plateaux anatoliens, attirés par sa position stratégique sur la route septentrionale, dite royale, de la Perse.

Divers autres peuples ont, depuis, paru sur la scène historique, détruisant systématiquement ce que leurs prédécesseurs y avaient bâti : c'est ainsi que le Grand Alexandre, les Romains. les Byzantins, les Perses, les Arabes (Haroun-al Rachid), les Croisés, les Seldjoukides, les Osmans et les Mongoles sous Tamerlan (Timour Lenk) ont occupé la ville et sa cita delle plus ou moins longtemps.

En 1923, à l'instigation du Gazi Mustapha Kemal (nommé actuellement Kamâl Ataturk), la petite bourgade d'Ankara fut choisie, pour des raisons politiques et stratégiques, comme capitale de la République, et des mesures lurent prises afin de créer les éléments, nécessaires pour la rendre digne de cette fonction.

Un concours international fut ouvert pour obtenir un plan d'ensemble de la ville future, reliant l'ancienne ville de 40.000 habitants à de nouveaux quartiers modernes et spacieux; de somptueux bâtiments furent érigés pour les ministères, les banques, les écoles, tous de style moderne et occidental, dont quelques-uns très réussis; des terrains furent réservés pour l'établissement d'un aéroport, d'un champ de courses, d'un stade, de parcs de récréation, etc.

L'amélioration des installations, nécessaires pour assurer l'une des fonctions essentielles d'une vie urbaine, l'alimentation en eau potable, eut également l'attention des autorités.

D'après un recensement très récent (celui du 3 novembre 1935), la ville d'Ankara possède 123.000 habitants. La citadelle, à la silhouette célèbre et caractéristique, est reproduite à la fig. 1 ; une partie de la nouvelle ville, avec l'ancienne ville et la forteresse au second plan, sont montrées à la fig. 2 .

\section{L'alimentation en eau potable.}

Les plus anciennes adductions d'eau d'Ankara probablement datent de l'époque romaine.

Les fouilles, exécutées lors de la pose de nouvelles conduites, ont révélé, pendant ces dernières années, des par-

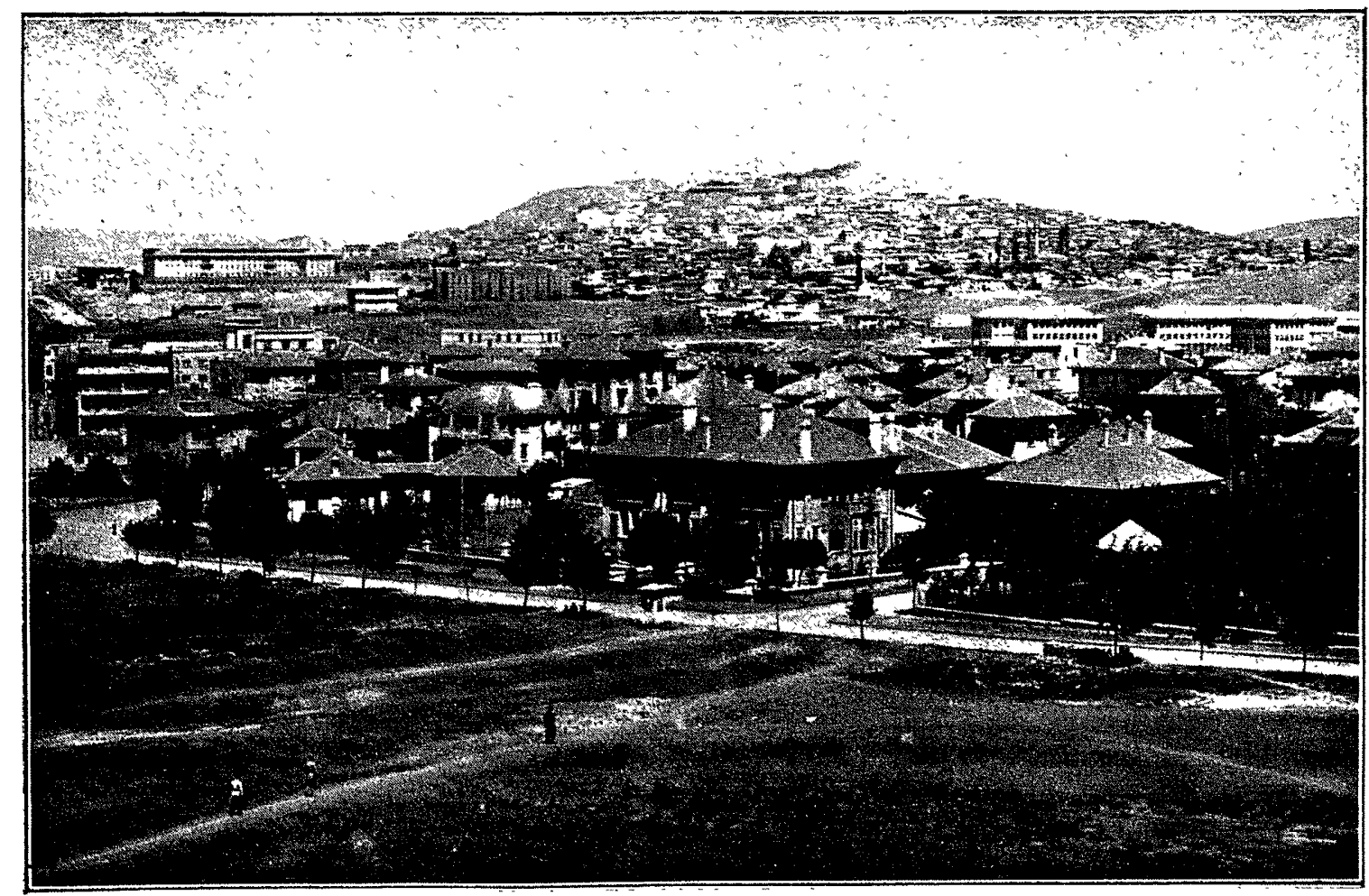

Fig. 2. - Vue d'une partie de la nouvelle ville; la ville ancienne au second plan. 
ties de conduites ou des tuyaux anciens de cinq types différents, dont nous reproduisons trois dans la fig. 3 . Ce sont, à gauche : des tuyaux en pierre (granit ou andésit) à paroi mince, enveloppés de mortier ; au centre: des tuyaux en grès, à paroi de $4 \mathrm{~cm}$, à emboîtement; à drorte :

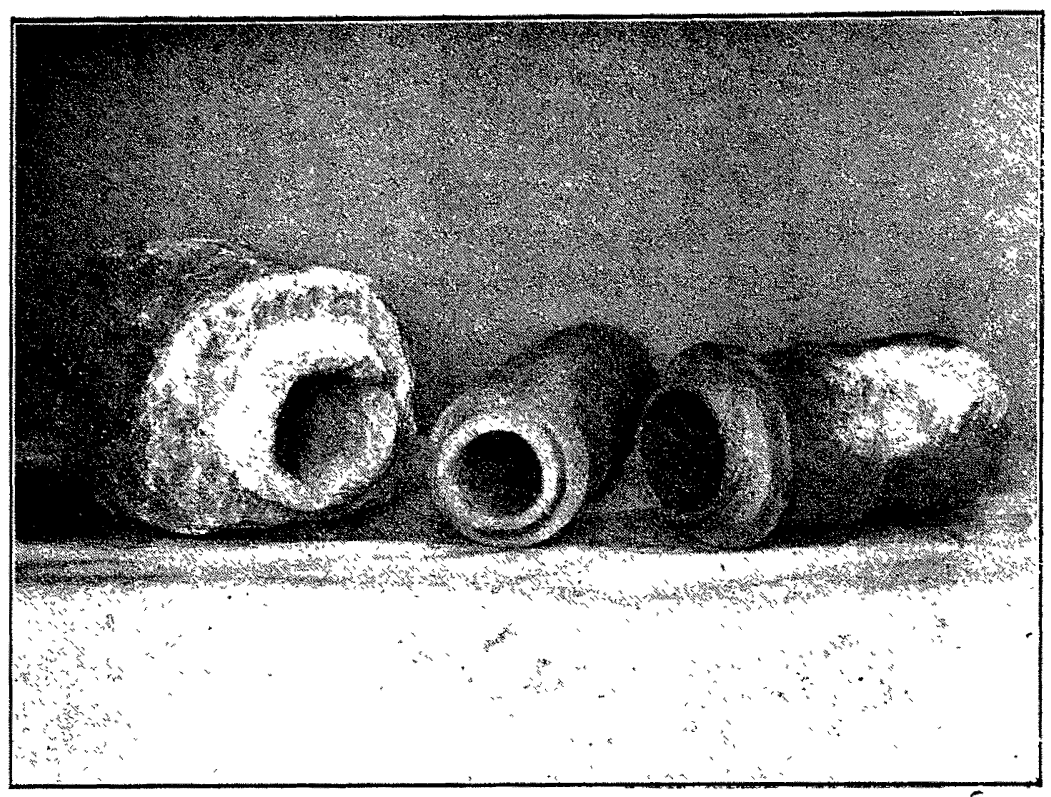

Fig. 3. - Trois types de tuyaux anciens, excavés lors de fouilles récentes.

des tuyaux en grès mince, de forme conique, enveloppés de mortier. Un quatrième type consistait en de grands blocs de pierre, de $50-60 \mathrm{~cm}$ carré, percés de trous cylindriques de $15-20 \mathrm{~cm}$, dont plusieurs ont été employés plus tard - par les Seldjoukides - dans la reconstruction des murs de la citadelle; des canaux en maçonnerie, enfin, couverts de grandes pierres plates, qui sont d'une origine plus récente, turque probablement.

Quant aux conduites en grès, les excavations lors du captage de la source Seki Pinar, à lamontagne dite Elma Dag (montagne des pommiers), en ont mises à jour quelques centaines de mètres des deux types (fig. 4). La situation de la source à une altitude plus élevée que celle de la cita delle, et le fait connu que les Romains avaient amené à celle-ci de l'eau courante, permettent, à notre avis, de déterminer ces tuyaux en grès comme romains, ainsi que les blocs de pierre percés, qui auraient été employés dans les sections à forte pression. La couleur du mortier, enveloppant les tuyaux en grès; supporterait, d'ailleurs, cette déduction, l'expérience dans les anciennes villes côtières de l'Asie Mineure ayant démontré que le mortier des Romains était blanc, celui des Byzantins contenait de la poussière de briques rouges, et celui des Turcs des particules de charbon.

Toutes ces vieilles conduites ayant, plus tard, tombé en ruines, c'était en 1890 que le Vali Abrdin Pacha fit construire deux canaux en maçonnerie, amenant ainsi en ville, l'eau de la source dite Hanim Pinar, dans la vallée de Kayas, et de deux sources dans l'Elma Dag (voir la fig. 5), tandis qu'en ville quelques conduites en fonte et en acier forgé furent posées, et reliées à des fontaines publiques.

Peu après qu'Ankara avait été choisie comme capitale, des crédits étaient mis à la dısposition de l'édilité par le gouvernement, en vue d'une amélioration de l'approvisionnement en eau potable, qui s'imposait.
Le projet, adopté pour ètre exécuté, consistait principalement en la construction d'un barrage souterrain dans la vallée de Kosunlar, la pose d'une conduite d'amenée en fonte, de $600 \mathrm{~mm}$. de diamètre intérieure et d'une longueur de 10 kilomètres, ainsi qu'un réseau de distribution et deux réservoirs.

Des difficultés d'ordre financier étaient cause, en 1928, d'une interruption temporaire des opérations, et les autorités municipales se voyaient forcées de commencer la distribution à l'aide de trois stations de pompage provisoires, installées à Kosunlar, à Sahne, et à Hanim Pinar, d'un réseau de distribution très réduil et d'un seul réservoir dans la nouvelle ville.

Le gouvernement central alors se décida de prendre l'affaire en mains, et de procéder conformément aux recommandations, exposées dans un rapport du Directeur Général des travaux hydrauliques, en date du $1 \mathrm{er}$ avril 1931. De nouveaux crédits furent alors votés, et l'exécution du programme confiée à une Commission gouvernementale, aux services de laquelle l'auteur de cet article entra commé ingénieur spécialiste.

Le rapport, dans ses grandes lignes, recommandait : l'achèvement du barrage souterrain de Kosunlar; l'exécution en forme définitive de la station de pompage de Hanim Pinar; l'élaboration d'un projet et l'exécution d'un réseau complet de distribution ; le captage de sources dans l'Elma Dag, et la pose de conduites d'amenée; enfin : recherches de nouvelles prises d'eau et exécution éventuelle des travaux nécessaires.

Le barrage souterrain de Kosunlar, que la fig. 6 montre en cours de construction, consiste en un mur en béton, d'une longueur de $96,5 \mathrm{~m}$., encastré dans le rocher, et coupant ainsi les couches aquifères des alluvions de la vallée. L'eau souterraine entre à travers plusieurs couches de gravier de dimensions différentes dans une galerie. épargnée dans le mur, et coule ensuite par un tunnel, d'une

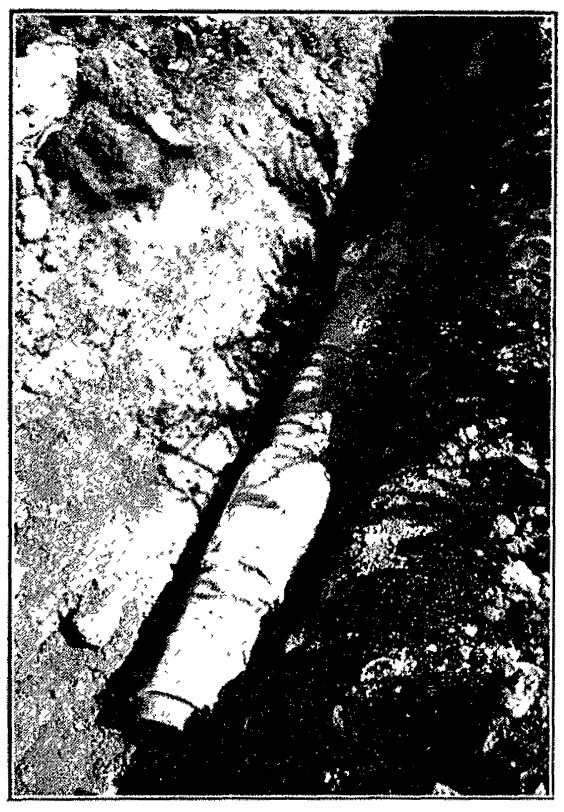

Fig. 4. - Conduite ancienne, probablement romaine, excavée près de la source Seki Pinard à l'Elma Dag (Montagne des pommiers). 
longueur de $400 \mathrm{~m}$ et la conduite en fonle de $600 \mathrm{~mm}$, mentionnée plus haut, à la ville.

Il est à remarquer qu'un barrage de ce type présuppose la possibilité d'un certain emmagasinement de l'eau souterraine; dans ce cas, cependant, cette faculté du sous-sol était relativement restreinte. Le barrage commande un bassin de précipitation de 40,8 kilomètres carrés, presque dépourvu de végétation; la précipitation annuelle y est de $200 \mathrm{~mm}$ d'amenée de $58 \mathrm{~km}$ environ. Les réservoirs, qui ont été exécutés principalement en béton armé, se trouvent à Koca Tep $\left(1.000 \mathrm{~m}^{3}\right)$, Hisar $\left(2.400 \mathrm{~m}^{3}\right)$, Bedestan $\left(3.500 \mathrm{~m}^{3}\right)$, Cankaya $\left(1.900 \mathrm{~m}^{3}\right)$, Cebeci $\left(430 \mathrm{~m}^{3}\right)$ et Kale $\left(180 \mathrm{~m}^{3}\right)$; voir la carte, fig. 5 .

$\Lambda$ l'usage de deux des zones les plus élevées des dispositifs spéciaux, nommés "Hydrostats", ont trouvé une application utile. Ces" machines, des béliers à pistons, utilisent

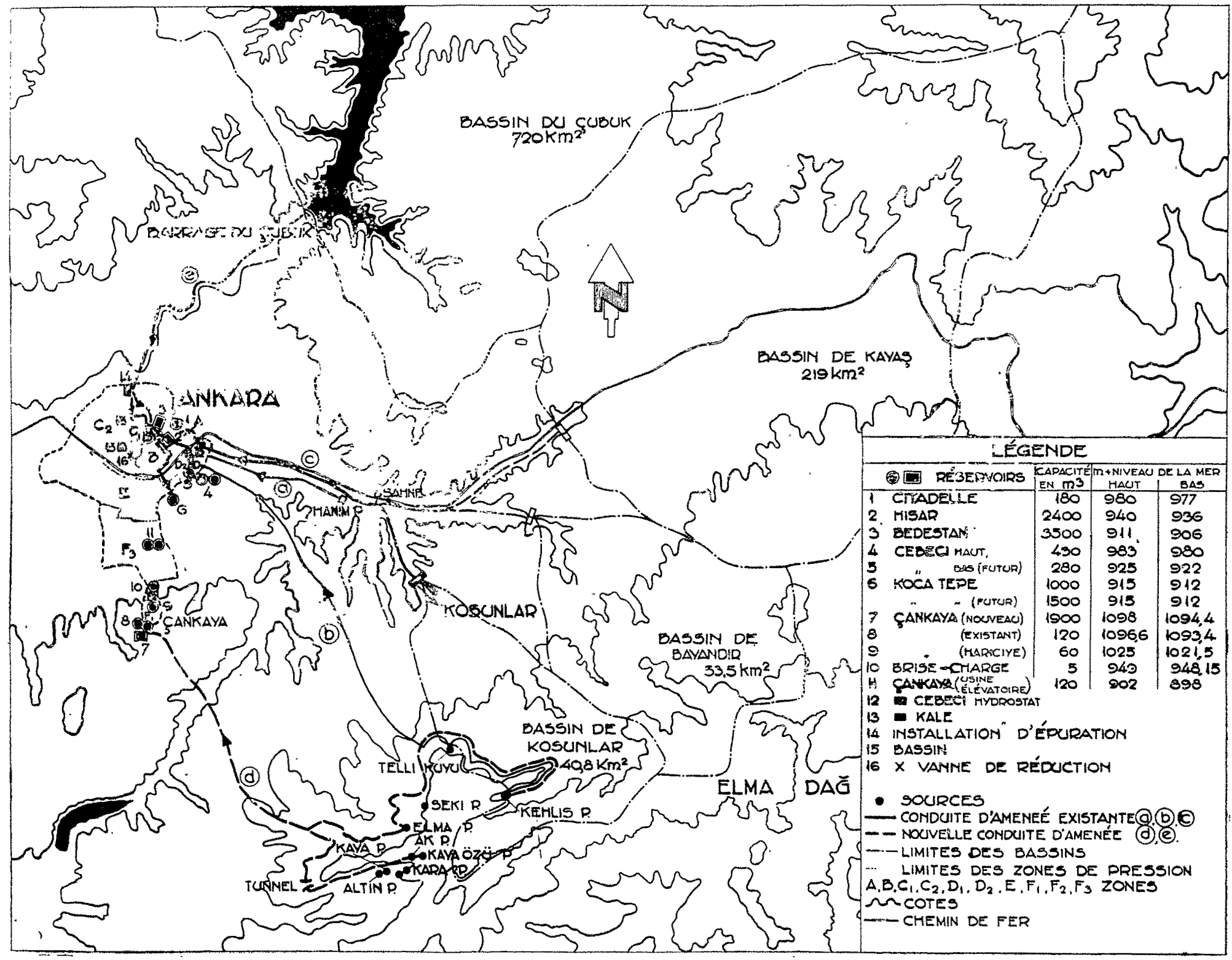

0
1

Fig. 5. - Carte des environs d'Ankara, montrant les adductions d'eau actuelle.

seulement ; l'évaporation atteint un chiffre dans les environs de $2.000 \mathrm{~mm}$.

Le réseau de distribution devait ètre adopté aux conduites existantes. Les grandes différences d'élévation dans les divers quartiers de la ville nécessitaient une division du réseau en dix zones de pression différente; sept ont été munies d'un réservoir, les autres ont été séparées entre elles par des vannes réductrices ou des chambres brise-charge.

La longueur totale des conduites principales, toutes exécutées en fonte, est de $200 \mathrm{~km}$, celle des conduites un surplus de pression dans la conduite-maîtresse comme force motrice pour refouler de l'eau à une cote plus élevée. L'une d'elles, celle pour la zone de la citadelle, a été désignée pour refouler 12 litres/sec, à une hauteur de 65 mètres ; l'autre, destinée à desservir la zone haute de Cebeci, et qui a été exécutée en trois unités, fait monter 14 litres/sec. à une altitude de 85 mètres au-dessus des machines.

En vue d'un contròle effectif de la marche du service, des compteurs-enregistreurs ont été installés à différents endroits, entre autres dans plusieurs réservoirs, ainsi que des transmetteurs automatiques du niveau d'eau et des téléphones privés, reliant les réservoirs au bureau central. 


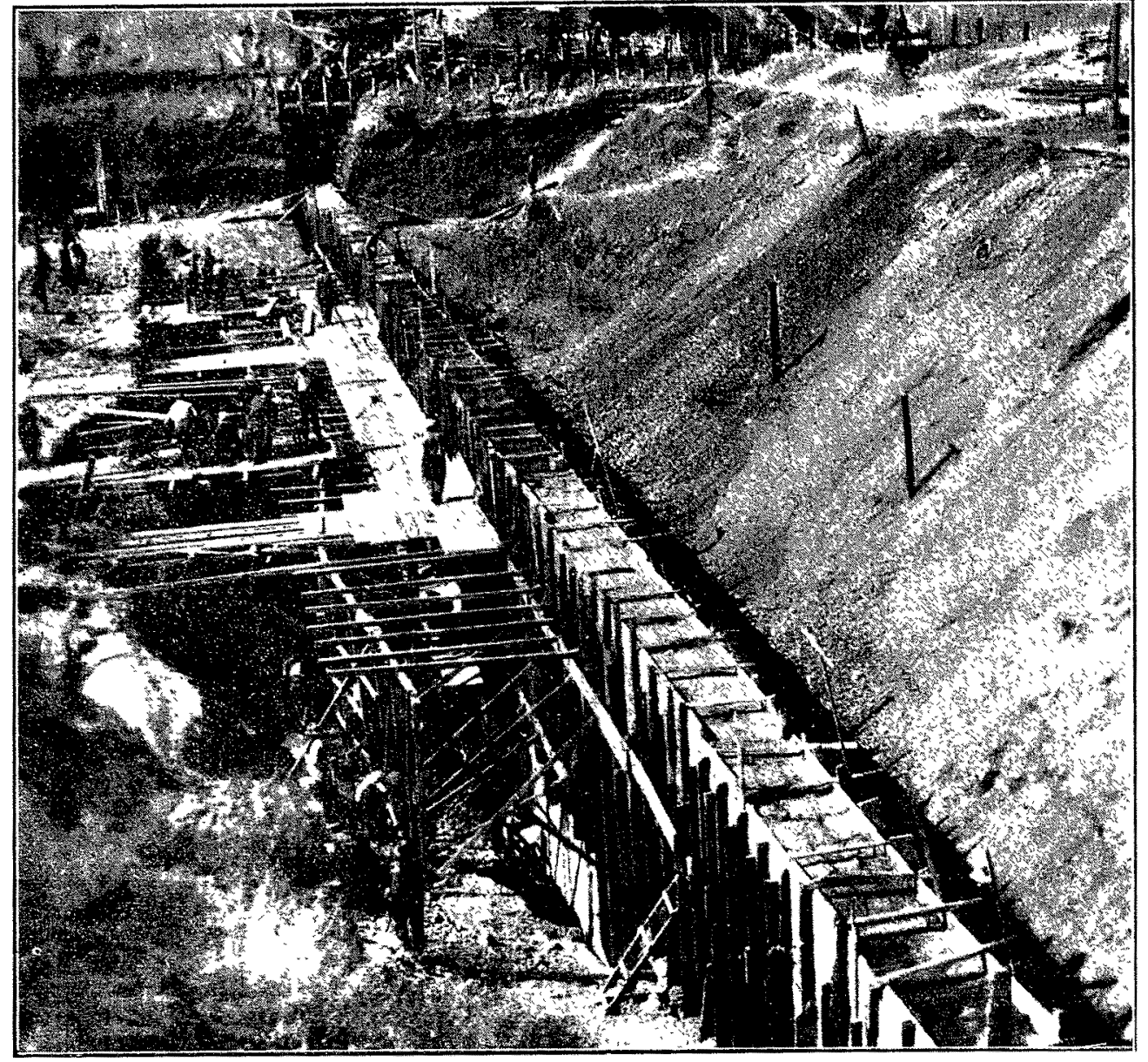

Fig. 6. - Le barrage souterrain de Kosunlar pendant la construction; du Kizil Irmak et de la Sakaria, furent examinées (voir la fig. 9).

Le projet du Çubuk proposait d'utiliser les eaux d'un barrage réservoir, qui se trouvait en cours de construction sous la direction de la Direction générale des travaux hydrauliques, à une distance de 11 kilomètres au N. E. de la ville. Ce barrage, dont le bassin de précipitation est de 720 kilomètres carrés, était destiné à femmagasiner les eaux de la petite rivièrs du Cubuk, principalement en vue d'irrigations à faire dans la vallée en aval de la ville.

Il se trouvait pourtant que les précipitations n'étaient pas de nature a garantir en toutes circonstances les quantités d'eau désirables ̀̀ cette fin ; les besoins en eau potable, cependant, pourraient, en toute probabilité, y être satisfait toujours.

Le barrage, dont nous montrons deux photographies dans les fig. 10 et 11, a été projeté pour former un réservoir de 12 millions de $\mathrm{m}^{3}$; 1 ll est du type, dit de gravité, en plan courbé, et a été exécuté exclusivement en béton.

Sa longueur développée est de 252 mètres; la crête se trouve à une hauteur de 25 mètres au-dessus du point le plus bas de la vallée ; la

A l'Elma Dag plusieurs sources ont été captées, et l'eau amenée par des conduites de gravité vers Cankaya, le quartier gouvernemental, au point le plus élevé duquel a été bâti le palais du Président de la République.

Les conduites, dont la longueur totale est de 35,5 kilomètres environ, comportent un tunnel de 145 mètres de longueur et cinq syphons, dont l'un, descendant dans une vallée de 200 mètres de profondeur, a été doublé. Ces conduites ont été exécutées exclusivement en fonte, le syphon double en tuyaux en fonte de haute pression. Les conduites ont été divisées en différentes sections par des brise-charges, de sorte que la pression normale ne dépasse en général 35 mètres.

Les opérations de creusement de la tranchée dans un tronçon de terrain difficile sont reproduites dans la fig. 7 ; les profils longitudinaux, des conduites sont indiqués dans la fig. 8 .

Après l'achèvement de ces travaux la station de refoulement au pied de la colline de Sankaya, qui élevait l'eau de quelque 200 mètres, pouvait être mise hors service, permettant de réaliser ainsi une économie assez considérable.

Des recherches dans plusieurs vallées des environs au moyen de forages, la détermination de la vitesse de l'eau souterraine selon la méthode de Slichter, et d'autres investigations, faites en vue d'examiner les possibilités de nouvelles prises d'eau dans la proximité de la ville, démontraient que les quantités nécessaires pour les besoins de la ville à l'avenir ne pourraient pas ainsi être obtenues.

Pour cette rason trois autres solutions, que nous indiquerons respectivement comme les projets du Çubuk, fondation devait, à cause de la nature du sous-sol, être descendue à certains endroits jusqu'à une profondeur de 58 mètres au-dessous de la crête.

Le projet du Kizil Irmak (Rivière rouge, le Halys des anciens) avait pour base l'uttlisation des eaux de la rivière

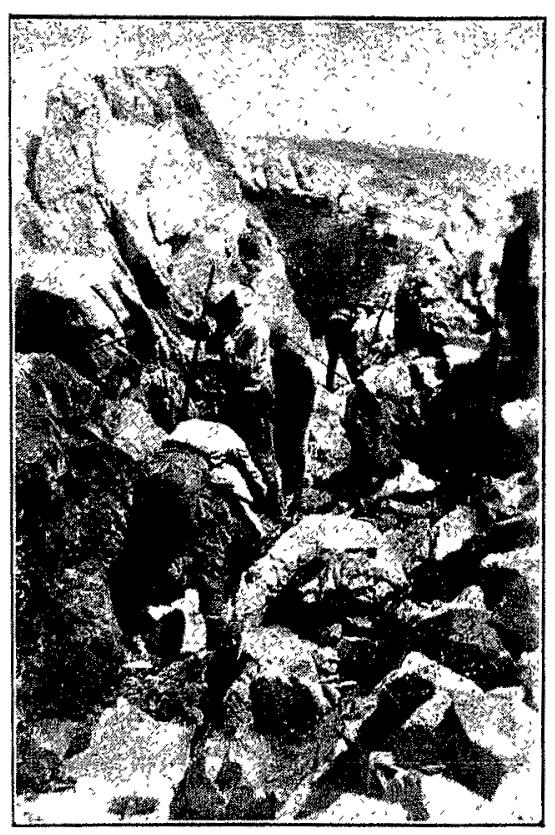

Fig. 7. - Tracé d'un tronçon difficile des conduites d'amenée à l'Elma Dag. 
de ce nom, dont la distance jusqu'à Ankara est de 56,3 kilomètres par la route directe.

L'eau, après avoir reçu sur place le traitement nécessaire, devrait être refoulée à travers une passe dans l'arête taine distance, en vue de diminuer la hauteur de refoulement, ne promettait pas d'éronomies.

Ce dernier projet présentait, en certains aspects, une ressemblance à celle qu'on a choisie pour résoudre le

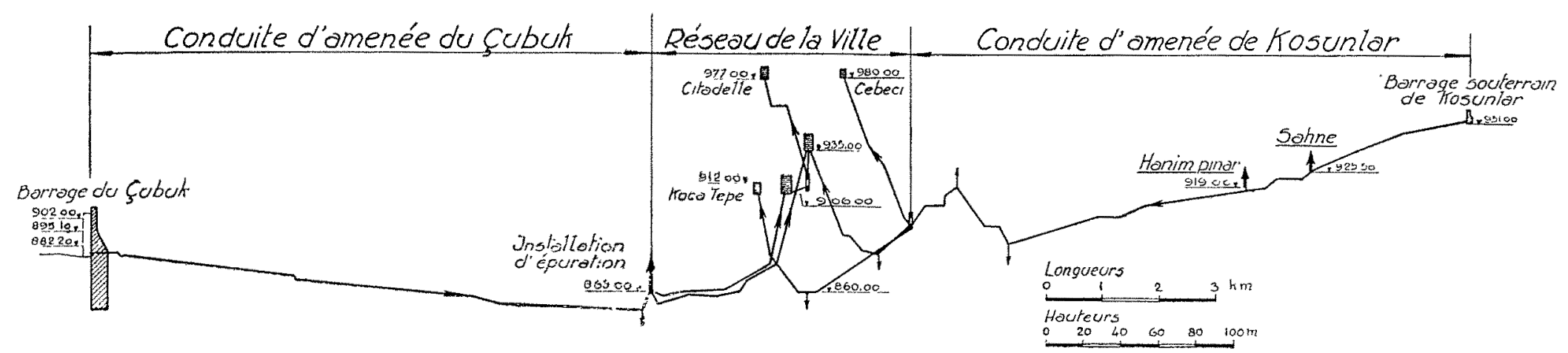

\begin{tabular}{|l|}
\hline \multicolumn{1}{|c|}{ Légende } \\
1 Stations de poimópoge \\
1 Hydrostots \\
I Sources \\
I Chombres brises-chorge \\
I Réservoirs \\
I Ventouses \\
I Déchorges
\end{tabular}

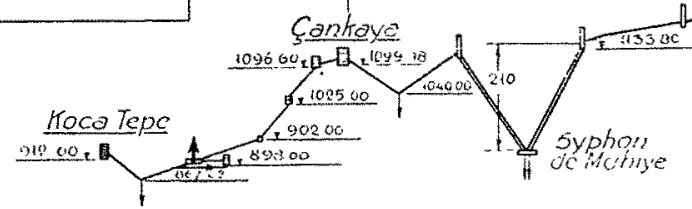

Conduites d'ámenée del'Elmo Dag

Fig. ১. - Profils longitudinaux des adaductions đ̆eau actueỉes.

de l'Elma Dag, une différence de niveau dé 550 inètres, qui nécessiterait l'utilisation d'au moins trois stations de pompage. L'énergie électrique nécessaire pourrait éventuellement être fournie par une usine hydro-électrique, à bâtir dans la rivière à une distance de 25 à 30 kilomètres en amont de la prise d'eau. La construction d'un tunnel sur une cer- problème de l'eau potable de la ville de Jérusalem, où, d'ailleurs, les distances et les hauteurs à surmonter sont plus considérables $(72 \mathrm{~km}$ et 914 mètres respectivement), et où l'on utilisera quatre stations de refoulement à moteurs Diesel ; l'eau, qu'on empruntera à la rivière souterraine de Ras-el-Ain, n'y subira d'autre traitement que la chloration.

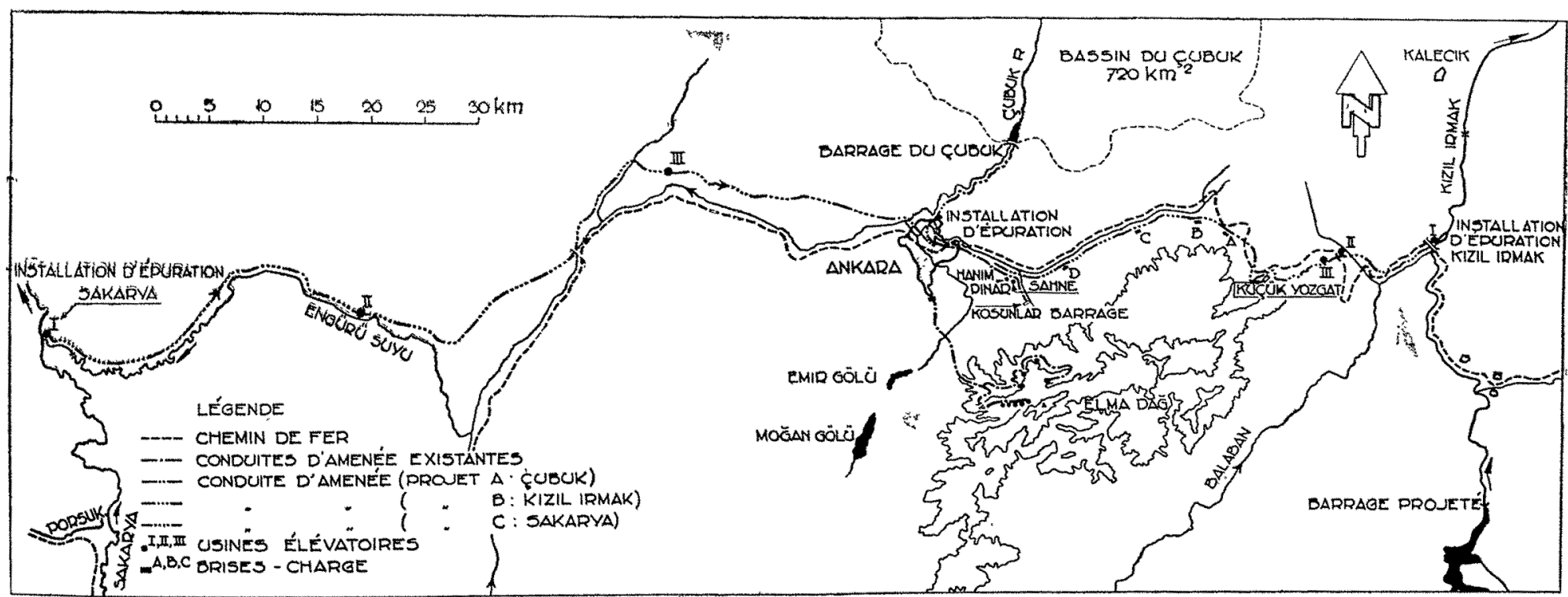

Fig. 9. - Trois projets d'alimentation en eau supplémentaire, dont le projet du Çubuk a été choisi et exécuté. 


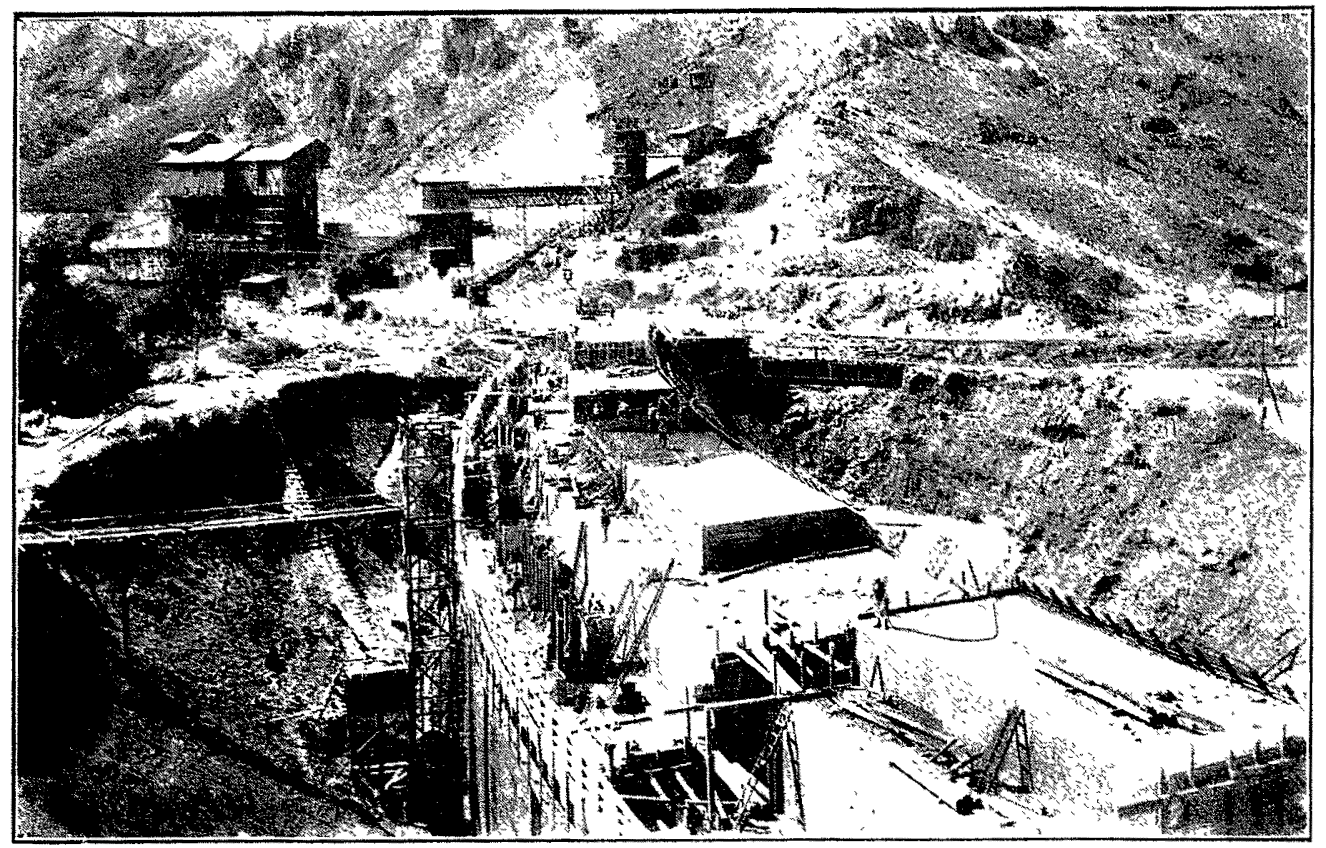

Des compteurs d'eau ont été installés systématiquement pour tous branchements privés, en vue de mettre fin au gaspillage, maintes fois constaté, et d'augmienter en même temps les revenus du service, qui suffisaient à peine à balancer les dépenses directes, le capital ayant été fourni à fonds perdus.

Après l'achèvement des divers travaux qui, à part des fournitures provenant de l'Europe, étaient exécutés principalement par des entrepreneurs et de la main d'œuvre du pays, les installations furent transférées successivement aux autorités municipales, et mises en service.

Fig. 10. - Le barrage du Gubuk en cours de construction.

Le projet de la Sakaria, enfin, proposalt d'utiliser l'eau de cette rivière, qui devait être transportée sur une distance de 101 kilomètres environ, et être refoulée sur une hauteur totale manométrique sensiblement la même que celle, nécessaire dans le projet du Kizil Irmah.

En vue de la désirabilité de prendre des mesures sans délai, et surtout pour des raisons d'ordre économique, le projet du Cubuk devait être recommandé et préféré sur les deux autres. Des crédits supplémentaires furent alors votés pour l'achèvement du barrage, l'exécution d'une conduite d'amenée, et, l'établissement des installations d'épuration nécessaires.

La conduite, d'un diamètre intérieur de $600 \mathrm{~m} / \mathrm{m}$, a été exécutée en acier, soudé électriquement, en tuyaux à emboîtement à plomb.

L'eau coule par gravité aux installations d'épuration, qui se trouvent directement en amont de la ville, et d'où une branche permet de transporter de l'eau brute aux fontaines d'un parc de récréation.

Après l'épuration, qui se compose d'aération, de dosage de sulphate d'alumine, de sédimentation, de filtration rapide et de chloration, éventuellement combinée avec de l'ammoniation, l'eau est refoulée dans deux différentes zones de la ville. Le pompage se fait à l'électricité ; une réserve à moteurs Diesel a été prévue. Le dosage du sulphate d'alumine peut être effectué aussi bien avant qu'aprés l'aération ; un "saut hydraulique " a été prévu pour assurer un bon mélange dans ce dernier cas.

L'eau, provenant des prises d'eau existantes, avait été dıstribuée, originalement, sans avoir subi aucun traitement. Son degré hydrotimétrique se trouvait entr 16 et 24 degrés français. Il apparût désirable, néanmoins, d'envisager toutes éventualités, et des mesures ont été prises, en conséquence, pour être à même de stériliser au chlore toute eau distribuée, si la nécessité se présentait,

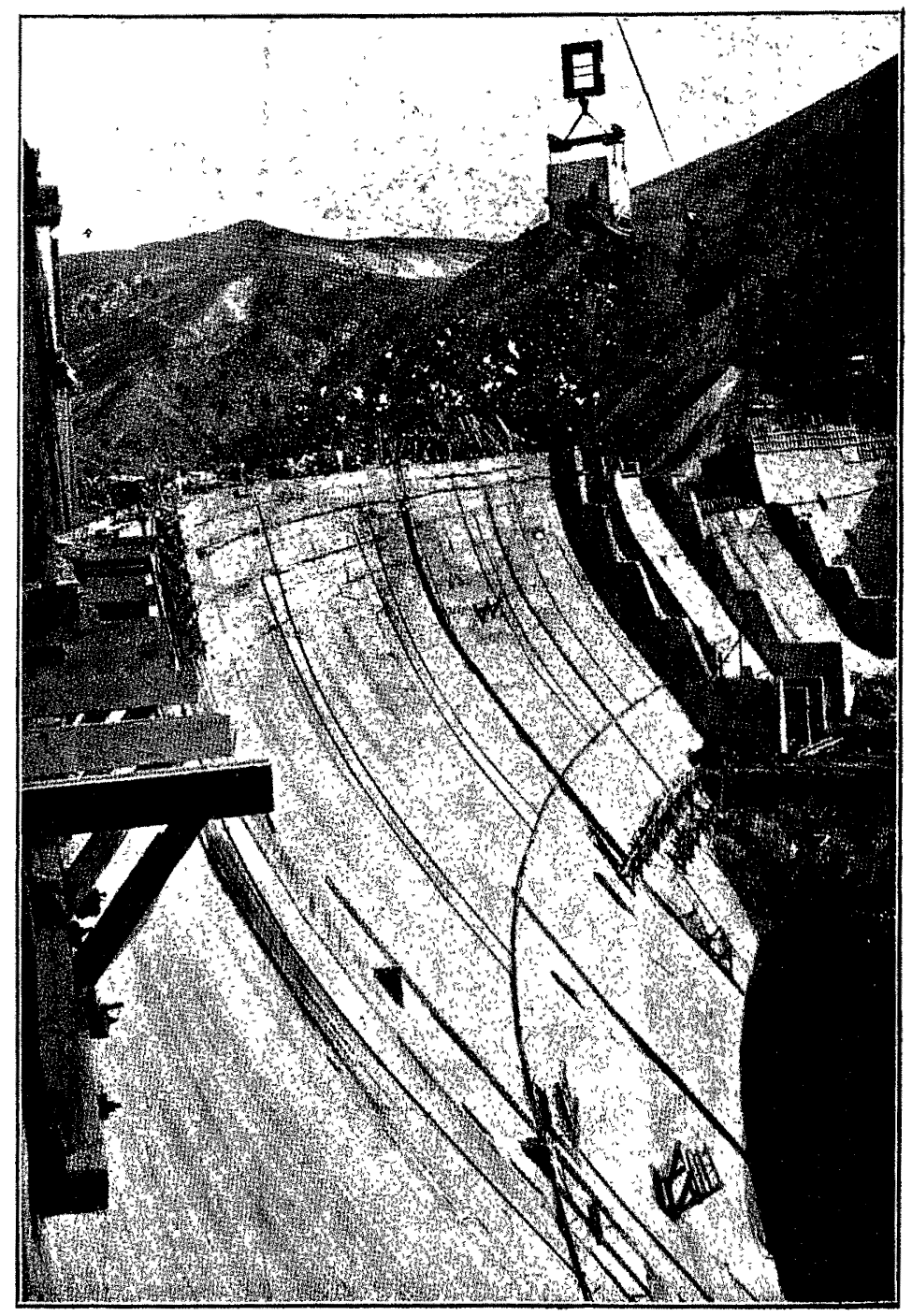

Fig. 11. - Le barrage de Gubuk péu avant l'achèvement. 